\title{
RESULTADOS DO TRANSPLANTE DE FÍGADO NA DOENÇA HEPÁTICA ALCOÓLICA
}

\author{
Mônica Beatriz PAROLIN' ${ }^{1}$, Júlio Cezar Uili COELHO ${ }^{1}$ e Mauro da IGREJA² \\ Maria Lúcia PEDROSO ${ }^{3}$, Anne Karoline GROTH ${ }^{2}$ e Carolina Gomes GONÇALVES ${ }^{4}$
}

RESUMO - Racional - O transplante de fígado é aceito como modalidade terapêutica efetiva nas doenças hepáticas avançadas, incluindo a cirrose alcoólica. Objetivo - Avaliar os resultados do transplante hepático em pacientes com cirrose alcoólica no Programa de Transplante Hepático do Hospital de Clínicas da Universidade Federal do Paraná, Curitiba, PR. Pacientes e Métodos - Análise retrospectiva dos pacientes com cirrose alcoólica submetidos a transplante hepático no Serviço de Transplante Hepático do Hospital de Clínicas da Universidade Federal do Paraná entre setembro de 1991 e janeiro de 2001. Todos os candidatos apresentavam período de abstinência de álcool $\geq 6$ meses antes do transplante. A identificação do consumo de álcool no período pós-transplante hepático baseou-se em: (1) informações fornecidas pelo paciente e/ou familiares, e/ou (2) anormalidades bioquímicas sugestivas de consumo abusivo de álcool associadas a anormalidades histológicas compatíveis com lesão pelo etanol. Resultados - Vinte adultos com cirrose alcoólica (19 homens e 1 mulher) com mediana de idade de 50 anos (29-61 anos) foram submetidos a transplante hepático, correspondendo a 13,8\% das indicações de transplante hepático em adultos. Em $30 \%$ dos pacientes (6/20) houve associação com hepatite viral crônica, e em 1 caso $(5 \%)$ presença de hepatocarcinoma. A maioria dos pacientes apresentava disfunção hepática grave no pré-transplante (75\% Child C). A mediana do tempo de abstinência pré-transplante foi de 24 meses ( 9 a 120 meses). A mediana do tempo de seguimento pós-transplante foi de 14 meses ( 1 a 66 meses). A sobrevida do paciente em 1 e 3 anos após o transplante hepático foi de $75 \%$ e $50 \%$, respectivamente. As principais complicações observadas foram: rejeição aguda $(\mathrm{n}=8 ; 40 \%)$, rejeição crônica $(\mathrm{n}=1 ; 5 \%)$, trombose da artéria hepática $(\mathrm{n}=3 ; 15 \%)$, complicações biliares $(\mathrm{n}=3 ; 15 \%)$ infecção por citomegalovírus $(\mathrm{n}=4 ; 20 \%)$, infecções bacterianas e fúngicas $(n=9 ; 45 \%)$. A incidência detectada de recidiva do consumo de álcool foi de $15 \%(3 / 20)$. Em um dos casos de recidiva o uso inadequado dos imunossupressores resultou em rejeição crônica com perda do enxerto. Conclusões - Transplante hepático na cirrose alcoólica associou-se a níveis satisfatórios de sobrevida a curto e médio prazo. No presente estudo a recidiva do consumo de álcool foi pequena, o que pode ser devido à falta de rastreamento adequado.

DESCRITORES - Transplante de fígado. Hepatopatias alcoólicas.

\footnotetext{
'Serviço de Transplante Hepático, Hospital de Clínicas da Universidade Federal do Paraná (HC-UFPR); ${ }^{2}$ Disciplina de Cirurgia do Aparelho Digestivo da UFPR; ${ }^{3}$ Ambulatório de Doença Hepática Alcoólica do HC-UFPR; ${ }^{4}$ Serviço Cirurgia Geral do Hospital Nossa Senhora das Graças, Curitiba, PR.

Endereço para correspondência: Dra. Mônica Beatriz Parolin - Rua Lamenha Lins, 2280 - 80220-80 - Curitiba, PR. e-mail:mbparolin@hotmail.com
} 


\section{INTRODUÇ̃̃OO}

O transplante de fígado $(\mathrm{TH})$ é aceito como terapia eficaz nas doenças hepáticas avançadas, incluindo a cirrose alcoólica. Embora a doença hepática alcoólica (DHA) figure entre as principais causas de cirrose hepática nos países ocidentais, até 1985 raramente tais pacientes eram beneficiados pelo recurso terapêutico do TH. O pessimismo em relação ao TH nos portadores de DHA baseava-se na crença de que os resultados do TH nesse grupo de pacientes eram inferiores aos obtidos em outras formas de doença hepática, aliado ao temor da recidiva do consumo de álcool após o transplante, resultando em dano sobre o enxerto ${ }^{(11)}$. A partir de 1985, estudos realizados em diferentes centros encontraram índices de sobrevida pós-transplante em pacientes com cirrose alcoólica semelhantes aos obtidos em pacientes com doença hepática não associada ao álcool. Em 1988, STARZL et al. ${ }^{(15)}$, avaliando 41 pacientes com cirrose alcoólica avançada submetidos a TH na Universidade de Pittsburgh, sob esquema de imunossupressão com ciclosporina, relataram sobrevida em 1 ano de $73,2 \%$ e em 3 anos de $68 \%$. Índices satisfatórios de sobrevida em 1 ano pós-TH em portadores de DHA, variando de $66 \%$ a $100 \%$, também foram documentados em outros centros americanos e europeus $^{(1,2,5,7,8,9,10,13,14,16)}$.

Se, por um lado, os resultados em termos de sobrevida em 1 e 5 anos pós-transplante nos pacientes com cirrose alcoólica se assemelham aos encontrados nos portadores de outras formas de doenças hepática, a possibilidade de recidiva do consumo do álcool após o TH causa preocupação e é ainda objeto de controvérsia e debate. A freqüência com que a recidiva de consumo de bebidas alcoólicas é detectada no acompanhamento pós-transplante é variável, dependendo em parte dos métodos empregados na avaliação e seleção dos candidatos na fase pré-transplante, bem como dos métodos empregados para tal rastreamento dessa complicação no póstransplante. Publicações relatando a experiência de diferentes centros transplantadores apontam para índices de recidiva em torno de $12 \%$ a $50 \%(2,6,7,8,9,10,11,13)$. A necessidade de intervalo de abstinência préTH tem sofrido reavaliações, mas a maioria dos centros de transplante adota mínimo de abstinência de pelo menos 6 meses antes do TH.

O objetivo do presente estudo é relatar os resultados obtidos com o transplante de fígado em pacientes com cirrose alcoólica no Serviço de Transplante Hepático do Hospital de Clínicas da Universidade Federal do Paraná, Curitiba, PR.

\section{PACIENTES E MÉTODOS}

Entre setembro de 1991 e janeiro de 2001, realizaram-se 152 transplantes hepáticos em adultos (idade $\geq 18$ anos) no Serviço de Transplante Hepático do Hospital de Clínicas da Universidade Federal do Paraná, Curitiba. Em 20 desses 152 transplantes a etiologia da doença hepática foi cirrose alcoólica, correspondendo a 13,8\% das indicações de TH em adultos no referido Serviço. O diagnóstico de cirrose alcoólica foi feito com base na história de consumo abusivo de álcool (60 g de etanol/dia para homens e $40 \mathrm{~g}$ de etanol/dia para mulheres por, pelo menos, 8 anos) na ausência de outras causas de doença hepática, achados laboratoriais e clínicos compatíveis, biopsia hepática compatível com cirrose alcoólica e achados do exame anatomopatológico do figado nativo retirado no transplante. Todos os pacientes foram submetidos a avaliação psicossocial na fase pré-transplante, sendo exigido período mínimo de abstinência de, pelo menos, 6 meses antes do TH.

As seguintes variáveis pré e pós-transplante foram coletadas na revisão dos prontuários médicos: idade na ocasião do transplante, sexo, grau de disfunção hepática pré-TH de acordo com a classificação de Child-Pugh, presença concomitante de hepatite viral crônica e/ou hepatocarcinoma, tempo de abstinência pré-transplante, sobrevida do paciente pós-transplante, ocorrência de complicações tais como rejeição do enxerto, infecções, complicações técnicas vasculares e biliares, necessidade de retransplante, causas de óbito. O consumo de qualquer quantidade de bebida alcoólica após o transplante foi considerado recidiva e baseou-se na coleta de informações contidas nos prontuários médicos além de: (1) informações fornecidas pelo paciente e/ou familiares através de contato telefônico e/ou pessoal; (2) anormalidades bioquímicas sugestivas de consumo abusivo de álcool (macrocitose e elevação da gama-glutamiltransferase), associadas a anormalidades histológicas compatíveis com lesão pelo etanol.

Até 1998, esquema de imunossupressão primária empregou prednisona, ciclosporina em microemulsão, e azatioprina. Desde 1999 o Serviço passou a adotar como esquema padrão na imunossupressão primária o tacrolimus associado à prednisona com retirada gradual do corticóide. O uso do micofenolato de mofetil foi reservado para casos especiais como resgate de episódios de rejeição e para redução da dose dos inibidores da calcineurina devido à instalação de efeitos adversos intoleráveis atribuídos a esses fármacos.

\section{Análise estatística}

Os resultados das variáveis contínuas foram expressos em mediana e variação, e das variáveis categóricas em percentagens. O programa computacional utilizado foi o software Statistic $6.0^{\circledR}$ para Microsoft Windows ${ }^{\circledR}$.

\section{RESULTADOS}

\section{Características pré-transplante}

O primeiro TH realizado para doença hepática alcoólica no Hospital de Clínicas da Universidade Federal do Paraná foi em março de 1995. 
Dezenove dos 20 pacientes (95\%) eram do sexo masculino, a mediana da idade do grupo na época do transplante foi 50 anos (29 a 61 anos). As principais características clínicas dos pacientes na fase prétransplante estão descritas na Tabela 1 . Setenta e cinco por cento (15/ 20) dos pacientes apresentavam disfunção hepática grave de acordo com a classificação de Child-Pugh (classe C). Em 6 dos 20 pacientes $(30 \%)$ foi constatada associação com hepatite viral crônica, enquanto hepatocarcinoma foi detectado em 1 paciente. A mediana do período de abstinência pré-TH foi 24 meses, variando de 9 a 120 meses (Tabela 1).

\section{Sobrevida e complicações pós-transplante}

Quatorze dos 20 pacientes (70\%) submetidos a TH por cirrose alcoólica encontravam-se vivos até janeiro de 2001. A sobrevida em 1 ano e 3 anos após o TH foi de $75 \%$ e $50 \%$, respectivamente. A mediana do tempo de sobrevida pós-TH dos pacientes avaliados, até a época do estudo, foi de 14 meses, variando de 1 a 66 meses. A sobrevida do enxerto hepático 1 e 3 anos após o transplante foi de $68,7 \%$ e 33,3\%, respectivamente. Os 14 pacientes vivos e em acompanhamento apresentam-se clinicamente bem e com boa função do enxerto, sendo que em 78,6\% deles (11/14) a base da imunossupressão é o tacrolimus, e nos demais a ciclosporina em microemulsão.

As principais complicações encontradas na amostra estudada estão sumarizadas na Tabela 2. A incidência de rejeição celular foi 40\% (8/ 20) e todos os episódios foram responsivos à pulsoterapia com metilprednisolona. Um único caso de rejeição crônica $(5 \%)$ foi constatado, em paciente masculino de 31 anos de idade, cerca de 2 anos após o TH. O quadro foi atribuído ao uso irregular de ciclosporina após recidiva do consumo de álcool, de acordo com informações fornecidas pelo próprio paciente e familiares. Apesar da administração de dois ciclos de pulsoterapia com metilprednisolona e posteriormente OKT3, instalou-se ductopenia, falência do enxerto e óbito.

TABELA 1 - Características clínicas em 20 pacientes com cirrose alcoólica submetidos a transplante hepático

\begin{tabular}{lc}
\hline Variáveis pré-transplante & Pacientes $(\mathbf{n}=\mathbf{2 0})$ \\
\hline Distribuição por sexo (masculino/feminino) & $19 / 1$ \\
Idade (anos) & $50(29-61)$ \\
Classe de Child-Pugh : A/B/C & $1 / 4 / 15$ \\
Doença hepática associada à & \\
cirrose alcoólica & 5 \\
Hepatite C & 1 \\
Hepatite B & 1 \\
Hepatocarcinoma & \\
Tempo de abstinência pré-transplante (meses) & $24(9-120)$ \\
\hline
\end{tabular}

Nota: Todos os dados estão expressos em mediana e variação
Em relação às complicações vasculares, foram registrados três casos de trombose de artéria hepática para os quais foi indicado retransplante. Um paciente faleceu enquanto aguardava o procedimento e os demais foram submetidos a cirurgia de retransplante, 8 e 17 meses após o TH. Dos dois pacientes retransplantados, um faleceu no pós-operatório imediato por não funcionamento primário do enxerto enquanto o outro encontra-se clinicamente bem e com boa função do enxerto, 2 anos após o retransplante.

Três pacientes (15\%) desenvolveram complicações biliares, estando a trombose da artéria hepática implicada em um dos casos. Conforme descrito na Tabela 2, as complicações biliares foram estenose isolada $(n=2)$ e estenose associada à fístula biliar $(n=1)$. O paciente apresentando estenose associada à fistula biliar foi submetido a derivação biliodigestiva (hepaticojejunoanastomose) com sucesso. Em um dos pacientes com estenose biliar diagnosticou-se a presença de trombose de artéria hepática sendo, então, submetido a transplante hepático. O outro caso de estenose biliar manifestou-se sob a forma de sepse biliar com rápida evolução para óbito a despeito de antibioticoterapia de amplo espectro.

Oito dos 20 pacientes apresentaram infecção bacteriana após o TH: infecção de parede $(n=2)$, infecção do líquido ascítico $(n=2)$, colangite $(\mathrm{n}=2)$, infecção do trato urinário $(\mathrm{n}=1)$ e gastroenterite $(\mathrm{n}$ $=1$ ). Houve apenas um caso de infecção fúngica invasiva (meningite criptocócica) que se instalou 2 meses após o transplante, de evolução rapidamente fatal e cujo diagnóstico foi firmado à necropsia. Quatro pacientes apresentaram reativação da infecção por cytomegalovirus, sendo tratados, com sucesso, com ganciclovir endovenoso.

$\mathrm{Na}$ amostra estudada, detectou-se um caso de carcinoma de palato em paciente do sexo masculino, tabagista crônico, 27 meses após o transplante. $\mathrm{O}$ tratamento adotado foi a ressecção cirúrgica associada à radioterapia. Atualmente o paciente apresenta-se clinicamente bem, com boa função do enxerto e sem evidência de recidiva tumoral.

TABELA 2 - Complicações técnicas e clínicas em 20 pacientes com cirrose alcoólica submetidos a transplante hepático

\begin{tabular}{lc}
\hline Complicações & número de pacientes (\%) \\
\hline Trombose da artéria hepática & $3(15)$ \\
Complicações biliares* & $3(15)$ \\
Rejeição celular aguda & $8(40)$ \\
Rejeição crônica & $1(5)$ \\
Reativação da infecção por & $4(20)$ \\
cytomegalovirus & \\
Infecções bacteriana & $8(40)$ \\
Infecção fúngica & $1(5)$ \\
Carcinoma de palato & $1(5)$
\end{tabular}

* Estenose, $\mathrm{n}=2$ (um associado à trombose da artéria hepática); estenose + fístula biliar, $\mathrm{n}=1$ 
O paciente com diagnóstico pré-transplante de hepatocarcinoma e com seguimento de 2 anos após o TH não apresenta indícios de recidiva tumoral.

\section{Causas de óbito}

Dos seis pacientes que evoluíram para óbito durante o período do estudo, cinco faleceram nos 3 primeiros meses após o TH. Em três dos cinco casos $(60 \%)$ de óbito precoce (até 3 meses após TH) a causa foi infecciosa: sepse pulmonar ( $14^{\circ}$ pós-operatório), meningite criptocócica em paciente com trombose de artéria hepática ( $72^{\mathrm{a}}$ pósoperatória) e sepse biliar ( $108^{\circ}$ pós-operatório). Nos outros dois pacientes as causas de óbito precoce foram sangramento incontrolável após retransplante por trombose de artéria hepática ( $2^{\circ}$ pós-operatório) e falência de múltiplos órgãos ( $34^{\mathrm{a}}$ pós-operatório). Óbito tardio ( 2 anos após o TH) ocorreu em um paciente com falência do enxerto, secundária à rejeição crônica ductopênica.

\section{Recidiva do consumo de álcool após o transplante}

Em 3 dos 20 pacientes (15\%) detectou-se consumo de bebidas alcoólicas após o transplante, segundo informação fornecida pelos pacientes $(n=2)$ ou familiares $(n=1)$. Um dos pacientes admitiu durante internamento hospitalar uso irregular da medicação imunossupressora a partir da época em que passou a consumir bebidas alcoólicas, em freqüência quase diária, o que resultou em rejeição crônica e óbito. Outro paciente, portador de cirrose alcoólica associada à infecção pelo vírus da hepatite $\mathrm{C}$, e com antecedentes de uso de drogas ilícitas, após receber alta hospitalar no oitavo dia de pósoperatório, não manteve mais contato com a equipe de transplante hepático que, através de busca ativa, foi informada pelos familiares que o mesmo voltara a consumir bebidas alcoólicas e fora a óbito no $14^{\circ}$ dia de pós-operatório por quadro de sepse pulmonar. O terceiro paciente, que evoluiu a óbito por sepse biliar secundária a estenose biliar, 108 dias após o TH, segundo informações fornecidas por seus familiares, teria consumido algumas doses de bebida alcoólica após o TH. Cabe salientar que em todos os casos obedeceu-se ao critério de abstinência de álcool e drogas ilícitas por, pelo menos, 6 meses antes do transplante.

\section{DISCUSSÃO}

A DHA representa a segunda principal indicação de TH na atualidade, sendo superada apenas pela hepatite $\mathrm{C}^{(4,12)}$. Antes do advento do transplante, não existia terapia específica para a DHA, com exceção da abstinência alcoólica. Embora esta medida seja o fundamento do tratamento da cirrose alcoólica, sabe-se que seu impacto na sobrevida de portadores de doença hepática avançada é limitado.
Nessas circunstâncias, o TH é a única opção que oferece real benefício em termos de sobrevida a longo prazo. O presente estudo, avaliando 20 adultos portadores de cirrose alcoólica e em abstinência de consumo de álcool por pelo menos 6 meses, encontrou sobrevida de 1 e 3 anos pós-TH de $75 \%$ e $50 \%$, respectivamente. Esses resultados de assemelham aos encontrados em portadores de outros formas de doença hepática em nosso Serviço (dados não publicados) bem como aos reportados em outros centros transplantadores ${ }^{(1,2,7,8,9,10,13,16)}$. Segundo a literatura, existem apenas mínimas diferenças quanto à sobrevida do paciente, sobrevida do enxerto e outras avaliações de saúde, quando comparados receptores com doença hepática alcoólica e o restante dos receptores do transplante hepático.

A inclusão da DHA nos programas de transplante hepático sempre sofreu resistência por parte da opinião pública e de segmentos da comunidade médica. O principal argumento utilizado era a grande probabilidade de que índices elevados de recidivismo diminuíssem a sobrevida a longo prazo quer pelo desenvolvimento de lesões graves no enxerto associadas ao etanol, ou devido a outros problemas médicos com uso inadequado de imunossupressores ou complicações associadas ao alcoolismo crônico. Na presente série a taxa detectada de recidiva, definida como consumo de qualquer quantidade de álcool após o transplante, foi da ordem de $15 \%$. Vale mencionar que, como os instrumentos utilizados para coleta de tal informação incluíam análise retrospectiva de prontuários médicos e, quando possível, entrevista ou contato telefônico com pacientes e familiares, é provável que os dados obtidos subestimem a real prevalência de recidivismo.

Muitos estudos a respeito da recidiva do consumo de álcool após o TH têm sido publicados na última década ${ }^{(2,6,7,8,9,10,11,13)}$. A maioria deles são estudos retrospectivos, com períodos relativamente curtos de acompanhamento (geralmente inferior a 5 anos) e número pequeno de pacientes. Entretanto, algumas conclusões coerentes podem ser extraídas desses estudos. Contestando os baixos índices de recidiva encontrados inicialmente por STARZL et al. ${ }^{(15)}$, provavelmente $30 \%$ a $50 \%$ dos receptores alcoolistas admitem ou são identificados como tendo consumido alguma quantidade de álcool nos 5 primeiros anos pós-transplante. Muitos dos episódios de consumo de álcool após o TH são eventos isolados, após os quais os pacientes restabelecem abstinência. Felizmente, o uso continuado ou abusivo é muito menos comum, afetando $10 \%$ ou menos dos receptores alcoolistas. TANG et al. ${ }^{(17)}$ sugeriram que o intervalo entre o transplante e o retorno ao consumo abusivo de álcool é pequeno, freqüentemente menor que 1 ano.

KUMAR et al.$^{(9)}$ relataram a taxa de $43 \%$ de recidiva nos pacientes que bebiam ativamente dentro do 6 meses que antecederam o TH, em contraste com a taxa de $6,7 \%$ em pacientes que permaneceram abstinentes por, pelo menos, 6 meses antes do transplante. Outros estudos também confirmaram a correlação entre a duração da abstinência pré-transplante e a recidiva ao alcoolismo subsequente ${ }^{(4)}$. 
BRAVATA et al. ${ }^{(3)}$ não encontraram diferença estatisticamente significativa na proporção de receptores de transplante com DHA e não-alcoólica em relação ao relato de consumo de álcool após o $\mathrm{TH}$ ( $4 \%$ vs. $5 \%$ ao $6^{\circ}$ mês e $17 \%$ vs. $16 \%$ ao $12^{\circ}$ mês pós-transplante). Entretanto, entre os pacientes que consumiam álcool após o transplante, receptores com doença hepática não-alcoólica eram mais propensos ao consumo moderado (quatro a sete doses/semana), enquanto aqueles com DHA eram mais sujeitos aos consumo excessivo de álcool ( $>7$ doses/semana). Dois dos três pacientes da presente série, apresentaram uso irregular do imunossupressor após a recidiva do consumo de álcool, um dos quais desenvolveu rejeição crônica tardia, evoluindo com perda do enxerto e óbito. Relatos sobre o uso inadequado do esquema imunossupressor são também encontrados na literatura ${ }^{(10,17)}$. Um dos pacientes que recidivou precocemente o consumo de álcool, apresentou sepse de origem pulmonar de evolução fatal.

LUCEY et al. ${ }^{(10)}$ e VAILLANT ${ }^{(18)}$ observaram que pacientes com consumo significativo de álcool após TH apresentam maior freqüência de problemas não-hepáticos, tais como pancreatite e pneumonia.

A análise das complicações técnicas e clínicas que ocorreram após o TH no grupo estudado, cuja mediana do tempo de seguimento pósTH foi de 14 meses, não mostrou índices diferentes dos referidos na literatura médica (Tabela 2). As infecções foram a causa mais comum de óbito após o transplante. Em dois dos seis pacientes que tiveram evolução fatal pode-se considerar que a recidiva do consumo de álcool colaborou para o óbito: um caso em que o uso inadequado dos imunossupressores resultou em rejeição crônica ductopênica e um caso no qual, por recidiva precoce, houve abandono do seguimento e do uso correto dos imunossupressores, havendo instalação de sepse pulmonar e óbito. A freqüência de rejeição celular aguda e crônica foi de $40 \%$ e $5 \%$, respectivamente. Vários estudos observaram que pacientes submetidos a transplante hepático devido a cirrose alcoólica apresentam menor freqüência de episódios de rejeição celular aguda do que pacientes que são submetidos a transplantes por outras etiologias. Tais dados podem refletir o estado parcial de supressão da imunidade celular em pacientes com DHA avançada ${ }^{(11)}$.

Os dados do presente estudo permitem concluir que pacientes com doença hepática alcoólica, desde que devidamente selecionados, apresentam índices de sobrevida pós-transplante semelhantes aos encontrados em outras causas de doença hepática. É recomendável que tratamentos de suporte para a dependência química sejam mantidos no seguimento pós-transplante visto que, a recidiva do consumo de álcool pode ter impacto negativo na sobrevida póstransplante.

Parolin MB, Coelho JCU, Igreja M, Pedroso ML, Groth AK, Gonçalves CG. Outcome of liver transplantation in patients with alcoholic liver disease. Arq Gastroenterol 2002;39(3):147-152.

ABSTRACT - Background - Liver transplantation is accepted as effective therapeutic option for end-stage liver disease, including alcoholic liver disease. Aim - To evaluate the outcome of liver transplantation for alcoholic liver disease in the Liver Transplantation Program at "Hospital de Clínicas" of the Federal University of Paraná, Curitiba, PR, Brazil. Patients and Methods - It was performed a retrospective study of the patients who underwent liver transplantation for alcoholic end-stage liver disease between September 1991 and January 2001 . The minimum abstinence period required was 6 months before liver transplantation. Identification of alcohol consumption after liver transplantation was determinated by information provided by patient or family and biochemical or histological anormalities. Results - Twenty patients underwent liver transplantation for alcoholic liver disease in the study period, $95 \%(19 / 20)$ were men and the median age was 50 years (29-61 years). Seventy-five percent of the patients $(15 / 20)$ had severe liver disfunction (Child C class) in the pre-transplant period. In six of them (30\%) there was association with viral hepatitis and in one, with hepatocarcinoma. Median abstinence period before liver transplantation was 24 months, varying from 9 to 120 months. One-year and 3-year survival rate were $75 \%$ and $50 \%$, respectively. The main complications were: acute cellular rejection $(40 \%)$, chronic rejection $(5 \%)$, hepatic artery thrombosis $(15 \%)$, biliary complications $(15 \%)$, bacterial or fungal infections $(45 \%)$, cytomegalovirus infection $(20 \%)$. Three patients returned to alcohol use after liver transplantation. Conclusion - The survival of patients who received liver transplant for alcoholic cirrhosis are satisfactory. In the present study there was a small index of alcohol use after liver transplantation.

HEADINGS - Liver transplantation. Liver diseases, alcoholic.

\section{REFERÊNCIAS BIBLIOGRÁFICAS}

1. Anand AC, Ferraz-Neto BH, Nightingale P, Mirza DF, White AC, McMaster P, Neuberger JM. Liver transplantation for alcoholic disease: evaluation of a selection protocol. Hepatology 1997;25:1478-84.

2. Bird GLA, O'Grady JG, Harvey FAH, Calne RY, Williams R. Liver transplantation in patients with alcoholic cirrhosis: selection criteria and rates of survival and relapse. Br Med J 1990;301:15-7.

3. Bravata DM, Olkin I, Barnato AE, Keeffe EB, Owens DK. Employment and alcoho use after liver transplantation for alcoholic and nonalcoholic liver disease: a systematic review. Liver Transplant 2001;7:191-203.
4. Burke A, Lucey MR. Liver transplantation for alcoholic liver disease. Clin Liver Dis 1998;2:839-50.

5. DiMartini A, Jain A, Irish W, Fitzgerald MG, Fung J. Outcome of liver transplantation in critically ill patients with alcoholic cirrhosis. Transplantation 1998;66:298-302.

6. Gerhardt TC, Goldstein RM, Urschel HC, Tripp LE, Levy MF, Husberg BS, Jennings LW, Gonwa TA, Klintmalm GB. Alcohol use following liver transplantation for alcoholic cirrhosis. Transplantation 1996;62:1060-3

7. Gish RG, Lee AH, Keeffe EB, Rome H, Concepcion W, Esquivel CO. Liver transplantation for patients with alcoholism and end-stage liver disease. Am J Gastroenterol 1993;88:1337-42. 
8. Knechtle SJ, Fleming MF, Barry KL, Steen D, Pirsch JD, Hafez GR, D'Alessandro AM, Reed A, Sollinger HW, Kalayoglu M, Belzer FO. Liver transplantation for alcoholic liver disease. Surgery 1992;112:694-701.

9. Kumar S, Stauber RE, Gavaler JS, Basista MH, Dindzans VJ, Schade RR, Rabinovitz M, Tarter RE, Gordon R, Starzl TE, van Thiel DH. Orthotopic liver transplantation for alcoholic liver disease. Hepatology 1990;11:159-64.

10. Lucey MR, Carr K, Beresford TP, Fisher LR, Shieck V, Brown KA, Campbell DA, Appelman HD. Alcohol use after liver transplantation in alcoholics: a clinical cohort follow-up study. Hepatology 1997;25:1223-7.

11. Lucey MR. Liver transplantation in the alcoholic disease. In: Maddrey WC, Schiff ER, Sorell MF, editors. Transplantation of the liver. Philadelphia: Lippincott Williams \& Wilkins; 2000. p.319-25.

12. Maddrey WC. Alcohol-induced liver disease. Clin Liv Dis 2000;4:116-31.

13. Osorio RW, Ascher NL, Avery M, Bacchetti P, Roberts JP, Lake JR. Predicting recidivism after orthotopic liver transplantation for alcoholic liver disease. Hepatology 1994;20:105-10.

14. Poynard T, Barthelemy P, Fratte S, Boudjema K, Doffoel M, Vamlemmens C, Miguet JP, Mantion G, Messner M, Launois B, Navau S, Chaput JC. Evaluation of efficacy of liver transplantation in alcoholic cirrhosis by a case-control study and simulated controls. Lancet 1994;344:502-7.

15. Starzl ET, Thiel DV, Tzakis AG, Iwatsuki S, Todo S, Marsh JW, Koneru B, Staschak S, Stieber A, Gordon RD. Orthotopic liver transplantation for alcoholic cirrhosis. JAMA 1988;260:2542-4.

16. Stefanini GF, Biselli M, Grazi GL, Iovine E, Moscatello MR, Marsigli L, Fosch FG, Caputo F, Mazziotti A, Gasbarrini G, Cavallari A. Orthotopic liver transplantation for alcoholic liver disease: rates of survival, complications and relapse. Hepatogastroenterology 1997;44:1356-9.

17. Tang H, Boulton R, Gunson B. Patterns of alcohol consumption after liver transplantation. Gut 1998;43:140-5.

18. Vaillant GE. The natural history of alcoholism and its relationship to liver transplantation in alcoholics - a clinical-cohort follow-up study. Hepatology 1997;25:1223-7. 